\title{
Progressive Interval Type-I Censored Life Test Plan for Rayleigh Distribution
}

\author{
Arun Kaushik \\ Banaras Hindu University, \\ Varanasi, India-221005
}

\author{
Mradula \\ BBA University, \\ Lucknow, India-226025
}

\begin{abstract}
In this paper, we have considered the problem of optimal inspection times for the progressive interval type-I censoring scheme where uncertainty in the process is governed by the two-parameter Rayleigh distribution. Here, we also introduced some optimality criterion and determined the optimum inspection times, accordingly. The effect of the number of inspections and choice of optimally spaced inspection times based on the asymptotic relative efficiencies of the maximum likelihood estimates of the parameters are also investigated. Further, we have discussed the optimal progressive type-I interval censoring plan when the inspection times and the expected proportions of total failures in the experiment are under control.
\end{abstract}

Keywords: progressive interval type-I, optimality criterion, inspection plan, Rayleigh distribution.

\section{Introduction}

The Rayleigh distribution is recognized to be a very useful distribution in the lifetime analysis and operations research for its mathematical simplicity and statistical flexibility. It has numerous application in the diverse areas such as health, agriculture, biology, engineering, and other sciences. Rayleigh (1880) and Siddiqui (1962) have introduced this model and discussed its various captivating properties. The inferential problems regarding considered model have been discussed by Sinha and Howlader (1983), Lalitha and Mishra (1996) and Abd-Elfattah, Hassan, and Ziedan (2006). The probability density function of the Rayleigh distribution with parameters $\gamma$ and $\sigma$ is given by

$$
f(y)=\frac{y-\gamma}{\sigma^{2}} e^{-\frac{(y-\gamma)^{2}}{2 \sigma^{2}}} ; \quad y \in[\gamma, \infty) ; \gamma \in(-\infty, \infty) ; \sigma \in(0, \infty)
$$

and the distribution function is given by

$$
F(y)=1-e^{-\frac{(y-\gamma)^{2}}{2 \sigma^{2}}} ; \quad y \in[\gamma, \infty) ; \gamma \in(-\infty, \infty) ; \sigma \in(0, \infty)
$$


Mousa and Al-Sagheer (2005) have discussed Bayesian prediction whereas Wu, Chen, and Chen (2006) have performed Bayesian inference. Mousa and Al-sagheer (2006) have conducted statistical inference for progressive type-II censored data from the Rayleigh distribution. Seo and Kang (2007) obtained the approximate MLEs based on progressive type-II censored data and Kim and Han (2009) estimated the scale parameter under general progressive censoring. Recently, Dey and Dey (2014) estimates the parameters for Rayleigh distribution under progressively Type-II censoring with binomial removal and Abdel-Hamid and Al-Hussaini (2014) provided the Bayesian prediction analysis for Type-II progressive-censored data from the Rayleigh distribution under the progressive-stress model.

In life-testing experiments, many times, it is more economical and practical to gauge observations as progressive interval type-I (PITI) censored data than to record their actual measurements because exact observations may not be possible (e.g., in medical experiments) or may be very costly (e.g., engineering experiments which contain precious items). PITI censoring is a combination of interval Type-I censoring and progressive censoring, proposed by Aggarwala (2001), which is having wide applications in clinical trials. For more details about PITI censoring readers may refer to Kaushik, Singh, and Singh (2015), Kaushik, Pandey, Maurya, Singh, and Singh (2017), Ng and Wang (2009), Chen and Lio (2010), Lio, Chen, and Tsai (2011), etc. In the PITI censored situations, a natural problem that may arise is to determine the associated inspection times appropriately before conducting the experiment to assess the parameter(s) of interest with the least possible reduction in efficiency as compared to the exactly observed situation. In this context, Lin, Chou, and Balakrishnan (2013) have developed some optimum inspection plan for log-normal distribution. For this purpose, they proposed the use of maximization of the determinant of the Fisher information matrix or minimization of the determinant of the variance-covariance matrix. A discussion on optimal grouping or monitoring times can be found in the works of Kulldorf (1961) based on the criterion of minimizing the asymptotic variance or maximizing the determinant of the expected Fisher information matrix of the maximum likelihood estimates (MLEs) of the parameters under the interval type-I censoring scheme. Further for related work on optimal inspections times for lifetime censored data one may refer to Lin, Wu, and Balakrishnan (2009) and Aggarwal (1984). Our goal here is to determine the optimally spaced(OS) inspection times for the PITI censoring scheme concerning the two-parameter Rayleigh distribution by proposing some additional optimality criteria.

This paper is systematized into five sections. In section 2, we have described the Fisher's information matrix and variance-covariance matrix in case of Rayleigh distribution for a PITI censored sample. Section 3 devoted to the criteria for choosing the OS and the optimal equally spaced (OES) inspection times. In section 4, we have performed a numerical study and provided the discussion based on the results obtained thereof. The effect of the number of inspections and the choice of inspection times based on the asymptotic relative efficiencies (AREs) and relative entropy under the OS inspection scheme are assessed. In the same way, OES and EP inspection schemes are compared with the OS inspection scheme. Further, the optimal PITI censoring plan when the inspection times and the expected proportions of total failures in the experiment are pre-fixed have also been discussed. Finally, concluding remarks have been given in section 5 .

\section{Expected Fisher information matrix}

Let us consider a PITI censored data $D=\left(d_{1}, d_{2}, \ldots, d_{m}\right)$ and $R=\left(r_{1}, r_{2}, \ldots, r_{m}\right)$ from Rayleigh distribution. It is necessary to mention here that the values $R=\left(r_{1}, r_{2}, \ldots, r_{m}\right)$ may be pre-specified as the proportion $p_{1}, p_{2}, \ldots, p_{m}\left(\right.$ with $\left.p_{m}=1\right)$ of the remaining live units consequently, the numbers of units remaining at times $t_{1}, t_{2}, \ldots, t_{m}$ are random variables. Hence, the log-likelihood function for considered distribution under PITI censoring can be 
written as

$$
\log L(\gamma, \sigma)=\sum_{i=1}^{m} d_{i} \ln \left(e^{-\frac{\left(t_{i-1}-\gamma\right)^{2}}{2 \sigma^{2}}}-e^{-\frac{\left(t_{i}-\gamma\right)^{2}}{2 \sigma^{2}}}\right)-\frac{r_{i}\left(t_{i}-\gamma\right)^{2}}{2 \sigma^{2}}
$$

To obtain the maximum likelihood estimates of parameters $\sigma$ and $\gamma$, one requires to maximize Eq. (3) simultaneously with respect to $\sigma$ and $\gamma$. It is noticed here that the simultaneous solution of likelihood equations is not achievable in explicit form. Therefore, one can use a suitable numerical method to obtain the maximum likelihood estimates of parameters. Further, the Fisher's information matrix of the likelihood is obtained as

$$
I(\gamma, \sigma)=\mathrm{E}\left[\begin{array}{ll}
-\frac{d^{2} \log L}{d \gamma^{2}} & -\frac{d^{2} \log L}{d \gamma d \sigma} \\
-\frac{d^{2} \log L}{d \sigma d \gamma} & -\frac{d^{2} \log L}{d \sigma^{2}}
\end{array}\right]_{(\hat{\gamma}, \hat{\sigma})},
$$

where,

$$
\begin{gathered}
\frac{d^{2} \log L}{d \gamma^{2}}=\frac{1}{\sigma^{2}} \sum_{i=1}^{m} d_{i} \frac{\left(\mathrm{e}^{-0.5 \tau_{i}{ }^{2}}-\tau_{i}{ }^{2} \mathrm{e}^{-0.5 \tau_{i}{ }^{2}}-\mathrm{e}^{-0.5 \tau_{i-1}^{2}}+\tau_{i-1}^{2} \mathrm{e}^{-0.5 \tau_{i-1}^{2}}\right)}{\left(\mathrm{e}^{-0.5 \tau_{i-1}^{2}}-\mathrm{e}^{-0.5 \tau_{i}^{2}}\right)} \\
-d_{i} \frac{\left(\tau_{i-1} \mathrm{e}^{-0.5 \tau_{i-1}{ }^{2}}-\tau_{i} \mathrm{e}^{-0.5 \tau_{i}^{2}}\right)^{2}}{\left(\mathrm{e}^{-0.5 \tau_{i-1}^{2}}-\mathrm{e}^{-0.5 \tau_{i}{ }^{2}}\right)^{2}}-r_{i} \\
\frac{d^{2} \log L}{d \sigma^{2}}=\frac{1}{\sigma^{2}} \sum_{i=1}^{m} d_{i} \frac{\left(2-\tau_{i}\right) \tau_{i}^{2} \mathrm{e}^{-0.5 \tau_{i}^{2}}-\left(2-\tau_{i-1}\right) \tau_{i-1}^{2} \mathrm{e}^{-0.5 \tau_{i-1}^{2}}}{\left(\mathrm{e}^{-0.5 \tau_{i-1}^{2}}-\mathrm{e}^{-0.5 \tau_{i}{ }^{2}}\right)} \\
-d_{i} \frac{\left(-\tau_{i} \mathrm{e}^{-0.5 \tau_{i}{ }^{2}}+\tau_{i-1} \mathrm{e}^{-0.5 \tau_{i-1}^{2}}\right)\left(-\tau_{i}^{2} \mathrm{e}^{-0.5 \tau_{i}{ }^{2}}+\tau_{i-1}^{2} \mathrm{e}^{-0.5 \tau_{i-1}^{2}}\right)}{\left(\mathrm{e}^{-0.5 \tau_{i-1}^{2}}-\mathrm{e}^{-0.5 \tau_{i}{ }^{2}}\right)^{2}}-2 r_{i} \tau_{i-1} \\
\frac{d^{2} \log L}{d \gamma d \sigma}=\frac{1}{\sigma^{2}} \sum_{i=1}^{m} d_{i} \frac{\left(3-\tau_{i}^{2}\right) \tau_{i}^{2} \mathrm{e}^{-0.5 \tau_{i}^{2}}-\left(3-\tau_{i-1}^{2}\right) \tau_{i-1}^{2} \mathrm{e}^{-0.5 \tau_{i-1}^{2}}}{\mathrm{e}^{-0.5 \tau_{i-1}^{2}}-\mathrm{e}^{-0.5 \tau_{i}{ }^{2}}} \\
-d_{i} \frac{\left(\tau_{i-1}^{2} \mathrm{e}^{-0.5 \tau_{i-1}^{2}}-\tau_{i}^{2} \mathrm{e}^{-0.5 \tau_{i}^{2}}\right)^{2}}{\left(\mathrm{e}^{\left.-0.5 \tau_{i-1}^{2}-\mathrm{e}^{-0.5 \tau_{i}{ }^{2}}\right)^{2}}-3 r_{i} \tau_{i-1}^{2},\right.}
\end{gathered}
$$

and $\tau_{i}=\frac{t_{i}-\gamma}{\sigma}, d_{i}=\left(n-\sum_{l=1}^{i-1} d_{l}-\sum_{l=0}^{i-1} r_{l}\right) \times\left(\frac{F\left(t_{i}\right)-F\left(t_{i-1}\right)}{1-F\left(t_{i-1}\right)}\right)$, and $r_{i}=p_{i} \times\left(n-\sum_{l=1}^{i} d_{l}-\sum_{l=0}^{i-1} r_{l}\right)$. Therefore, the expected asymptotic variance-covariance matrix of the MLEs is

$$
\operatorname{Cov}(\gamma, \sigma)=-I^{-1}(\gamma, \sigma)=\left[\begin{array}{ll}
V_{11} & V_{12} \\
V_{21} & V_{22}
\end{array}\right]=V(\text { say })
$$

\section{Optimal inspection plan}

We have observed here that, generally the removals in the PITI censoring scheme are not in the control of the experimenter. Thus, we are left with optimization of the inspection plan 
only. That is $t_{1}, t_{2}, \ldots, t_{m}$ are to be chosen in accordance with an optimality criterion. Some optimality criteria used in this context are given in the following subsections:

\subsection{Optimality criterion based on the Fisher information matrix}

This criterion was suggested by Lin et al. (2009) to obtain the optimal choices of inspection times. According to them, the inspection times $t_{1}, t_{2}, \ldots, t_{m}$ are to be chosen so as to maximize the determinant of the Fisher information matrix given in Eq. (4) i.e.

$$
\max _{t_{1}<t_{2}<\cdots<t_{k}}|I(\cdot)| .
$$

\subsection{Optimality criterion based on generalized asymptotic variance (GAV)}

The determinant of the inverse of Fisher's information matrix is called as generalized asymptotic variance (see Bai, Kim, and Chun 1993). Ismail (2015) has suggested the use of GAV as the criterion to plan the inspection times. Therefore, the inspection times $t_{1}, t_{2}, \ldots, t_{k}$ are to be chosen such that GAV is minimized i.e.

$$
\min _{t_{1}<t_{2}<\cdots<t_{k}} G A V(\cdot)
$$

\subsection{Proposed optimality criterion based on Shannon entropy}

Shannon entropy provides the amount of information contained in the observed likelihood. Consequently, we propose to use it for the optimal choice of the inspection time $t_{1}, t_{2}, \ldots, t_{m}$. Thus, the resulting criterion is to choose the inspection times $t_{1}, t_{2}, \ldots, t_{m}$ which maximizing the Shannon entropy, i.e., the values of $t_{1}, t_{2}, \ldots, t_{m}$ are to be determined by

$$
\max _{t_{1}<t_{2}<\cdots<t_{m}} \mathbb{H}(\cdot),
$$

where, $\mathbb{H}$ is the Shannon entropy for PITI censored data obtained in the following Eq. (12).

$$
\begin{aligned}
\mathbb{H}(\underline{D})= & -\mathrm{E}\left[\ln f_{\underline{D}}\left(d_{1}, d_{2}, \ldots, d_{m}\right)\right] \\
= & \sum_{d_{1}=0}^{n} \sum_{d_{2}=0}^{n_{2}^{*}} \cdots \sum_{d_{m}=0}^{n_{m}^{*}} f_{\underline{D}}\left(d_{1}, d_{2}, \ldots, d_{m}\right) \times \ln f_{\underline{D}}\left(d_{1}, d_{2}, \ldots, d_{m}\right) \\
= & \sum_{d_{1}=0}^{n} \sum_{d_{2}=0}^{n_{2}^{*}} \cdots \sum_{d_{m}=0}^{n_{m}^{*}}\left\{\sum_{i=1}^{m} \ln \left(\begin{array}{c}
n_{i}^{*} \\
d_{i}
\end{array}\right)\right. \\
& +d_{i} \ln \left[F_{X}\left(t_{i}\right)-F_{X}\left(t_{i-1}\right)\right]-n_{i}^{*} \ln \left[1-F_{X}\left(t_{i-1}\right)\right] \\
& \left.+\left(n_{i}^{*}-d_{i}\right) \ln \left[1-F_{X}\left(t_{i}\right)\right]\right\} \times \prod_{i=1}^{m}\left(\begin{array}{c}
n_{i}^{*} \\
d_{i}
\end{array}\right) \\
& \times\left[\frac{F_{X}\left(t_{i}\right)-F_{X}\left(t_{i-1}\right)}{1-F_{X}\left(t_{i-1}\right)}\right]^{d_{i}}\left[\frac{1-F_{X}\left(t_{i}\right)}{1-F_{X}\left(t_{i-1}\right)}\right]^{n_{i}^{*}-d_{i}},
\end{aligned}
$$

where $n_{1}^{*}=n$ and $n_{i}^{*}=n-\sum_{l=1}^{i-1}\left(d_{l}-r_{l}\right)$ for $i=2, \ldots, m$.

\subsection{Optimality criterion based on the variance of the estimate of some specific population characteristic}

In many realistic circumstances, one may often be interested in some specific characteristics of the population which is a function of the parameters. Then one would be interested in minimization of the variance of the estimate of the characteristic under interest rather than 
minimization of the variance-covariance matrix of the estimate of the parameters. For example, one may be interested in getting a precise estimate of the population mean rather than the individual estimate of the parameters. The population mean $\mu$ for Rayleigh distribution is

$$
\mu=\gamma+\sigma \sqrt{\frac{\pi}{2}}
$$

Let $\hat{\mu}$ be the MLE of mean lifetime. Then, our proposed criterion is to choose $t_{1}, t_{2}, \ldots t_{m}$ so as to minimize $\operatorname{var}(\hat{\mu})$

$$
\min _{t_{1}<t_{2}<\cdots<t_{k}} \operatorname{Var}(\hat{\mu}) .
$$

Following Kamakura and Yanagimoto (1989) and using the delta method, the asymptotic variance of $\hat{\mu}$ will be

$$
\left[\begin{array}{ll}
\mu_{\gamma} & \mu_{\sigma}
\end{array}\right] I^{-1}(\gamma, \sigma)\left[\begin{array}{l}
\mu_{\gamma} \\
\mu_{\sigma}
\end{array}\right]
$$

where,

$$
\begin{aligned}
& \mu_{\gamma}=\frac{\partial}{\partial \gamma} \mu(\sigma, \gamma)=1 \\
& \mu_{\sigma}=\frac{\partial}{\partial \sigma} \mu(\sigma, \gamma)=\sqrt{\frac{\pi}{2}} .
\end{aligned}
$$

The above-stated criteria can be used for the choice of the inspection plan to design the experiment. The $t_{1}<t_{2}<\cdots<t_{k}$ obtained by using any of the aforesaid criteria will be called as optimal spaced (OS) inspection plan. However, if one is interested in keeping the inspection time equally spaced i.e. $t_{i}=i t, i=1,2, \ldots, m$, where $t$ to be chosen such that it maximizes the determinant of the Fisher information matrix i.e.

$$
\max _{t} \mathbb{H}(\cdot)
$$

Such inspection plan may be called optimal equal spaced (OES) inspection plan. For optimization, we propose to use the Simulated Annealing algorithm (see Corana, Marchesi, Martini, and Ridella (1987)). Further, we fix termination point $t_{m}$ at the $95 \%$ quantile of considered distribution and then we calculate the inspections time following the way that every interval having an equal probability of occurring an event called as equal-probability (EP) spaced inspection plan.

\section{Numerical result and discussion}

In this section, we explore the optimal choice for inspection times utilizing a numerical study. The numerical study has been performed using different values of the number of inspections $m$ and sample size $n$ based on the optimality criteria discussed in Sections 3.1, 3.2, 3.3 and 3.4, respectively. First, we computed the inspection times by using the transformation $\tau_{i}=\frac{t_{i}-\gamma}{\sigma}$. It is noted here that the optimal choice of $\tau_{i}$ will become independent of population parameters $\left(\gamma\right.$ and $\sigma$ ) for all the considered criterion mentioned above. Given $p_{1}=\cdots=p_{m-1}=0$, we compute the OS inspection times for each of the optimality criteria, in the form $\tau_{i}$ 's for $m=2,3, \ldots, 10$. The results are presented in Table 1 , where last three columns of this table show asymptotic relative efficiencies (AREs) of the estimates. Here, ARE is defined as the ratio of the asymptotic variance of parameters in the complete sample case to the that of in the PTIT censored case. Thus, we obtained the AREs of the MLEs of $\gamma$ and $\sigma$ as $A R E(\hat{\gamma})=\frac{2 / n * \sigma^{2}(1-\pi / 4)}{V_{11}}, \quad A R E(\hat{\sigma})=\frac{\sigma^{2} / 4 n}{V_{22}}$, respectively. It may be noted that $V_{11} / \sigma^{2}$ and $V_{22} / \sigma^{2}$ are functions of $\tau_{i}$ 's only (i.e. independent of $\gamma$ and $\sigma$ ).

Table 1 contains the optimal inspection times under considered criterion along with respective AREs. It is noted here that the $A R E(\hat{\mu})$ is highest for criterion discussed in section 3.4, 
Table 1: The inspection times under the different optimal criteria in terms of $\tau_{i}=\frac{t_{i}-\gamma}{\sigma}$.

\begin{tabular}{|c|c|c|c|c|c|c|c|c|c|c|c|c|c|}
\hline \multicolumn{14}{|c|}{ Optimality Criterion based on Shannon Entropy } \\
\hline $\mathrm{m}$ & $\tau_{1}$ & $\tau_{2}$ & $\tau_{3}$ & $\tau_{4}$ & $\tau_{5}$ & $\tau_{6}$ & $\tau_{7}$ & $\tau_{8}$ & $\tau_{9}$ & $\tau_{10}$ & $A R E(\hat{\mu})$ & $A R E(\hat{\gamma})$ & $A R E(\hat{\sigma})$ \\
\hline 2 & 1.1664 & 2.9371 & & & & & & & & & 0.7153 & 0.7315 & 0.5575 \\
\hline 3 & 0.8922 & 1.4623 & 2.9402 & & & & & & & & 0.8360 & 0.8624 & 0.6437 \\
\hline 4 & 0.7528 & 1.1663 & 1.6418 & 2.9444 & & & & & & & 0.8706 & 0.9023 & 0.7632 \\
\hline 5 & 0.6636 & 1.0030 & 1.3407 & 1.7682 & 2.9791 & & & & & & 0.9111 & 0.9309 & 0.8211 \\
\hline 6 & 0.6004 & 0.8948 & 1.1687 & 1.4685 & 1.8664 & 3.0258 & & & & & 0.9357 & 0.9543 & 0.8634 \\
\hline 7 & 0.5527 & 0.8162 & 1.0519 & 1.2930 & 1.5695 & 1.9472 & 3.0858 & & & & 0.9524 & 0.9572 & 0.8967 \\
\hline 8 & 0.5149 & 0.7555 & 0.9653 & 1.1716 & 1.3924 & 1.6528 & 2.0161 & 3.1550 & & & 0.9607 & 0.9670 & 0.9282 \\
\hline 9 & 0.4840 & 0.7068 & 0.8975 & 1.0803 & 1.2682 & 1.4750 & 1.7236 & 2.0760 & 3.2313 & & 0.9651 & 0.9754 & 0.9317 \\
\hline 10 & 0.4581 & 0.6666 & 0.8427 & 1.0082 & 1.1742 & 1.3495 & 1.5460 & 1.7856 & 2.1301 & 3.3362 & 0.9790 & 0.9848 & 0.9494 \\
\hline \multicolumn{14}{|c|}{ Optimality Criterion based on $\operatorname{var}(\hat{\mu})$} \\
\hline $\mathrm{m}$ & $\tau_{1}$ & $\tau_{2}$ & $\tau_{3}$ & $\tau_{4}$ & $\tau_{5}$ & $\tau_{6}$ & $\tau_{7}$ & $\tau_{8}$ & $\tau_{9}$ & $\tau_{10}$ & $A R E(\hat{\mu})$ & $A R E(\hat{\gamma})$ & $A R E(\hat{\sigma})$ \\
\hline 2 & 1.0283 & 2.5788 & & & & & & & & & 0.7223 & 0.6434 & 0.631 \\
\hline 3 & 0.7862 & 1.2896 & 2.5834 & & & & & & & & 0.8537 & 0.7777 & 0.6972 \\
\hline 4 & 0.6638 & 1.0278 & 1.4622 & 2.6228 & & & & & & & 0.9003 & 0.8574 & 0.8011 \\
\hline 5 & 0.5849 & 0.8833 & 1.1936 & 1.5752 & 2.6530 & & & & & & 0.9253 & 0.9051 & 0.8311 \\
\hline 6 & 0.5281 & 0.7887 & 1.0400 & 1.3084 & 1.6619 & 2.7217 & & & & & 0.9437 & 0.9236 & 0.8752 \\
\hline 7 & 0.4874 & 0.7192 & 0.9371 & 1.1520 & 1.3974 & 1.7516 & 2.7759 & & & & 0.9511 & 0.9516 & 0.8963 \\
\hline 8 & 0.4528 & 0.6654 & 0.8591 & 1.0428 & 1.2402 & 1.4865 & 1.8136 & 3.1221 & & & 0.9684 & 0.954 & 0.924 \\
\hline 9 & 0.4262 & 0.6225 & 0.7989 & 0.9622 & 1.1287 & 1.3272 & 1.5500 & 2.0544 & 3.1985 & & 0.9806 & 0.9695 & 0.9338 \\
\hline 10 & 0.4031 & 0.5874 & 0.7503 & 0.8977 & 1.0453 & 1.2135 & 1.3913 & 1.7676 & 2.1084 & 3.6024 & 0.9865 & 0.9708 & 0.9421 \\
\hline \multicolumn{14}{|c|}{ Optimality Criterion based on Fisher information matrix } \\
\hline $\mathrm{m}$ & $\tau_{1}$ & $\tau_{2}$ & $\tau_{3}$ & $\tau_{4}$ & $\tau_{5}$ & $\tau_{6}$ & $\tau_{7}$ & $\tau_{8}$ & $\tau_{9}$ & $\tau_{10}$ & $A R E(\hat{\mu})$ & $A R E(\hat{\gamma})$ & $A R E(\hat{\sigma})$ \\
\hline 2 & 1.149941 & 2.8865 & & & & & & & & & 0.714 & 0.6995 & 0.5927 \\
\hline 3 & 0.875579 & 1.4341 & 2.8746 & & & & & & & & 0.8254 & 0.862 & 0.6572 \\
\hline 4 & 0.747316 & 1.1446 & 1.6301 & 2.9240 & & & & & & & 0.8527 & 0.8911 & 0.782 \\
\hline 5 & 0.651242 & 0.9862 & 1.3315 & 1.7513 & 2.9576 & & & & & & 0.8928 & 0.9299 & 0.8189 \\
\hline 6 & 0.594122 & 0.8801 & 1.1617 & 1.4550 & 1.8539 & 3.0309 & & & & & 0.9170 & 0.9451 & 0.8637 \\
\hline 7 & 0.551476 & 0.8021 & 1.0474 & 1.2895 & 1.5551 & 1.9558 & 3.0897 & & & & 0.9261 & 0.9598 & 0.8934 \\
\hline 8 & 0.509954 & 0.7442 & 0.9586 & 1.1617 & 1.3869 & 1.6596 & 2.0182 & 3.4726 & & & 0.9486 & 0.9685 & 0.9186 \\
\hline 9 & 0.482187 & 0.6932 & 0.8887 & 1.0779 & 1.2604 & 1.4821 & 1.7276 & 2.2847 & 3.55571 & & 0.957 & 0.9748 & 0.931 \\
\hline 10 & 0.458261 & 0.6536 & 0.8405 & 1.0068 & 1.1674 & 1.3573 & 1.5501 & 1.9731 & 2.34324 & 4.0126 & 0.9622 & 0.9793 & 0.9466 \\
\hline \multicolumn{14}{|c|}{ Optimality Criterion based on GAV } \\
\hline $\mathrm{m}$ & $\tau_{1}$ & $\tau_{2}$ & $\tau_{3}$ & $\tau_{4}$ & $\tau_{5}$ & $\tau_{6}$ & $\tau_{7}$ & $\tau_{8}$ & $\tau_{9}$ & $\tau_{10}$ & $A R E(\hat{\mu})$ & $A R E(\hat{\gamma})$ & $A R E(\hat{\sigma})$ \\
\hline 2 & 1.1418 & 2.8670 & & & & & & & & & 0.7109 & 0.6882 & 0.6591 \\
\hline 3 & 0.8728 & 1.4317 & 2.8697 & & & & & & & & 0.8191 & 0.7814 & 0.7015 \\
\hline 4 & 0.7364 & 1.1422 & 1.6252 & 2.9136 & & & & & & & 0.8437 & 0.8815 & 0.8283 \\
\hline 5 & 0.6498 & 0.9821 & 1.3262 & 1.7503 & 2.9490 & & & & & & 0.8731 & 0.9238 & 0.8514 \\
\hline 6 & 0.5874 & 0.8754 & 1.1563 & 1.4535 & 1.8467 & 3.0253 & & & & & 0.9135 & 0.938 & 0.8781 \\
\hline 7 & 0.5403 & 0.7993 & 1.0407 & 1.2798 & 1.5532 & 1.9458 & 3.0843 & & & & 0.9356 & 0.9558 & 0.9085 \\
\hline 8 & 0.5044 & 0.7391 & 0.9542 & 1.1588 & 1.3775 & 1.6512 & 2.0157 & 3.4692 & & & 0.9486 & 0.9631 & 0.9305 \\
\hline 9 & 0.4736 & 0.6912 & 0.8883 & 1.0689 & 1.2554 & 1.4740 & 1.7220 & 2.2826 & 3.5529 & & 0.9525 & 0.9721 & 0.945 \\
\hline 10 & 0.4484 & 0.6525 & 0.8341 & 0.9970 & 1.1614 & 1.3487 & 1.5459 & 1.9639 & 2.3425 & 4.0020 & 0.9761 & 0.9868 & 0.9639 \\
\hline
\end{tabular}

$A R E(\hat{\gamma})$ is highest for criterion described in Section 3.3 and $A R E(\hat{\sigma})$ is highest for criterion given in Section 3.2 among others for all the considered choices of $m$. From an extensive numerical study, that we carried out here, it has been revealed that using any of these three optimality criteria will lead to similar results in terms of efficiency. Therefore, we shall primarily report the results based on the optimality criterion discussed in Section 3.3 in the subsequent paragraphs.

Table 2 presents the optimal length of the inspection interval for choosing the OES inspection times when $m=2,3, \ldots, 9,10,15,20$ and $p_{1}=p_{2}=\cdots=p_{m-1}=0$. Similarly, results were also obtained for the estimation of the OS inspection plan under other censoring schemes. From Tables 1 and 2, we can see that as $m$ increases the AREs increases under all the considered criterion and for large $m$ AREs tend to 1. It is interesting to note here that for the estimation of $\gamma$ the choice of optimum censoring time leads to consistently higher ARE than that for the estimation of $\sigma$ in the considered cases. It may further be seen from the table that the performance of the estimates of $\gamma$ and $\sigma$ under PITI censoring will still be reasonably good if the number of inspections is chosen to be at least 5 and preferably 8 or more. For a comparison of the OS inspection scheme with the OES and EP inspection schemes based on $A R E(\gamma), A R E(\sigma), A R E(\mu)$ and Entropy respectively, we calculated the AREs, for different choices of $m$ when $p_{1}=\cdots=p_{m-1}=0$ and the results are summarized in Table 3 . It can 
Table 2: The optimal length of the inspection interval in terms of $\tau=\frac{t-\gamma}{\sigma}$ for OES inspection times using different optimality criterion when $p_{1}=\cdots=p_{m-1}=0$

\begin{tabular}{lrrrrrrrrrr}
\hline Criterian & $m$ & 2 & 3 & 4 & 5 & 6 & 7 & 8 & 15 & 20 \\
\hline Max. Entropy & 1.4505 & 0.9424 & 0.7382 & 0.5937 & 0.5033 & 0.4302 & 0.3863 & 0.2939 & 0.2112 \\
Min. GAV & 1.4846 & 0.9425 & 0.7352 & 0.6034 & 0.5267 & 0.4340 & 0.4046 & 0.3144 & 0.2491 \\
Min. Var $(\hat{\mu})$ & 1.4736 & 0.9667 & 0.7361 & 0.5958 & 0.5043 & 0.4408 & 0.3944 & 0.2990 & 0.2336 \\
Max. FIM & 1.4868 & 0.9821 & 0.7220 & 0.6046 & 0.5253 & 0.4627 & 0.3952 & 0.3078 & 0.2352 \\
\hline
\end{tabular}

Table 3: A comparison of the three inspection schemes based on asymptotic relative efficiencies and relative entropy

\begin{tabular}{lrrrrrrrrrr}
\hline$A R E$ & $m$ & 2 & 3 & 4 & 5 & 6 & 7 & 8 & 15 & 20 \\
\hline$A R E(\hat{\gamma})$ & & & & & & & & & \\
OS & 0.6995 & 0.8619 & 0.8910 & 0.9299 & 0.9450 & 0.9598 & 0.9684 & 0.9919 & 0.9972 \\
OES & 0.6763 & 0.8428 & 0.8459 & 0.8777 & 0.8941 & 0.9343 & 0.9605 & 0.9895 & 0.9928 \\
EP & 0.6727 & 0.8323 & 0.8397 & 0.8567 & 0.8829 & 0.9191 & 0.9526 & 0.9835 & 0.9902 \\
$A R E(\hat{\sigma})$ & & & & & & & & & \\
OS & 0.5927 & 0.6572 & 0.7819 & 0.8188 & 0.8636 & 0.8934 & 0.9185 & 0.9447 & 0.9631 \\
OES & 0.4856 & 0.6231 & 0.7499 & 0.7997 & 0.8408 & 0.8796 & 0.8803 & 0.9257 & 0.9481 \\
EP & 0.4843 & 0.5855 & 0.6860 & 0.7551 & 0.8123 & 0.8414 & 0.8581 & 0.9049 & 0.9458 \\
ARE $(\hat{\mu})$ & & & & & & & & & \\
OS & 0.7140 & 0.8254 & 0.8527 & 0.8927 & 0.9170 & 0.9461 & 0.9586 & 0.9573 & 0.9727 \\
OES & 0.6769 & 0.7970 & 0.8418 & 0.8792 & 0.9069 & 0.9171 & 0.9288 & 0.9435 & 0.9673 \\
EP & 0.6396 & 0.7673 & 0.8250 & 0.8412 & 0.8802 & 0.9054 & 0.9114 & 0.9394 & 0.9591 \\
Relative Entropy & & & & & & & & & \\
OS & 0.6472 & 0.8040 & 0.8134 & 0.8249 & 0.8718 & 0.8797 & 0.9381 & 0.9761 & 0.9888 \\
OES & 0.5977 & 0.7851 & 0.7916 & 0.8092 & 0.8619 & 0.8679 & 0.9286 & 0.9551 & 0.9728 \\
EP & 0.5667 & 0.7103 & 0.7892 & 0.7901 & 0.8444 & 0.8664 & 0.9047 & 0.9428 & 0.9545 \\
\hline
\end{tabular}

be seen easily from the table that the ARE for OS inspection plan is highest and for EP plan it is least in all the cases. The AREs for OES lies in between the AREs under OS and OES plans. Thus, on the basis of this, we may say that for the Rayleigh distribution, the OS inspection scheme is more suitable as it provides the maximum ARE as compared to other schemes irrespective of the parameter under deliberation.

To study the effect of variation in the values of $m$ and $n$ on the relative entropy we considered a number of values for $m$ and $n$ and taking an arbitrary choice for $p_{i}^{\prime} s$ as $p_{1}=p_{2}=\cdots=$ $p_{m-1}=0$. The results obtained are presented in Table 4 . It may be seen from the table that the relative entropy increases as $m$ or $n$ increases, but the increment in relative entropy is higher due to increase in $m$ as compared to that of increase in $n$.

We have noted above that AREs of the estimates for an optimum choice of the inspection time depends on the parameter to be estimated. Suppose that we are interested in the estimation of both parameters then neither the value of $A R E(\gamma)$ nor the value of $A R E(\sigma)$ can provide us the overall performance of the two estimates. Therefore, we need a single quantity

Table 4: The values of relative entropies for varying $m$ and $n$, when $p_{1}=\cdots=p_{m-1}=0$

\begin{tabular}{|c|c|c|c|c|c|c|}
\hline$m$ & 30 & 40 & 50 & 100 & 150 & 200 \\
\hline 2 & 0.6552 & 0.6924 & 0.6991 & 0.6996 & 0.7064 & 0.7546 \\
\hline 3 & 0.7028 & 0.7123 & 0.7265 & 0.7418 & 0.7527 & 0.7720 \\
\hline 4 & 0.7380 & 0.7458 & 0.7551 & 0.7666 & 0.7822 & 0.7968 \\
\hline 5 & 0.7587 & 0.7656 & 0.7765 & 0.7998 & 0.8158 & 0.8392 \\
\hline 6 & 0.7638 & 0.7829 & 0.8023 & 0.8406 & 0.8479 & 0.8723 \\
\hline 7 & 0.7890 & 0.8082 & 0.8145 & 0.8463 & 0.8795 & 0.8864 \\
\hline 8 & 0.8363 & 0.8456 & 0.8469 & 0.8564 & 0.8854 & 0.9123 \\
\hline 9 & 0.8497 & 0.8550 & 0.8792 & 0.8975 & 0.9316 & 0.9484 \\
\hline 10 & 0.8875 & 0.8955 & 0.8986 & 0.9327 & 0.9717 & 0.9816 \\
\hline
\end{tabular}


Table 5: The values of $\lambda A R E(\hat{\gamma})+(1-\lambda) A R E(\hat{\sigma}), \lambda \in(0,1)$, when $p_{1}=\cdots=p_{m-1}=0$

\begin{tabular}{|c|c|c|c|c|c|c|c|c|c|}
\hline$m$ & 0.1 & 0.2 & 0.3 & 0.4 & 0.5 & 0.6 & 0.7 & 0.8 & 0.9 \\
\hline 2 & 0.5514 & 0.5842 & 0.5892 & 0.5904 & 0.5923 & 0.5967 & 0.6050 & 0.6143 & 0.6490 \\
\hline 3 & 0.5737 & 0.6001 & 0.6093 & 0.6208 & 0.6298 & 0.6400 & 0.6515 & 0.6609 & 0.6684 \\
\hline 4 & 0.5995 & 0.6400 & 0.6413 & 0.6492 & 0.6538 & 0.6650 & 0.6812 & 0.6886 & 0.6944 \\
\hline 5 & 0.6351 & 0.6548 & 0.6654 & 0.6710 & 0.6744 & 0.6966 & 0.7120 & 0.7289 & 0.7354 \\
\hline 6 & 0.6521 & 0.6618 & 0.6801 & 0.7016 & 0.7116 & 0.7344 & 0.7459 & 0.7597 & 0.7640 \\
\hline 7 & 0.6804 & 0.6871 & 0.7007 & 0.7108 & 0.7363 & 0.7405 & 0.7794 & 0.7807 & 0.7841 \\
\hline 8 & 0.7230 & 0.7249 & 0.7394 & 0.7414 & 0.7432 & 0.7463 & 0.7811 & 0.7966 & 0.8069 \\
\hline 9 & 0.7396 & 0.7496 & 0.7512 & 0.7750 & 0.7800 & 0.7890 & 0.8258 & 0.8325 & 0.8364 \\
\hline 10 & 0.7463 & 0.7794 & 0.7884 & 0.7984 & 0.8070 & 0.8282 & 0.8629 & 0.8711 & 0.8805 \\
\hline
\end{tabular}

to measure the over all performance of the estimates. Therefore, a convex combination of $A R E(\gamma)$ and $A R E(\sigma)$ is a simple way to assess the relative efficiency of the two estimates considered simultaneously. Some convex combinations of these two AREs, viz., $\lambda A R E(\gamma)+$ $(1-\lambda) A R E(\sigma), \lambda \in[0,1]$ are presented in Table 5. The values of $A R E(\gamma)$ and $A R E(\sigma)$ are taken from Table 1 for those rows in which optimality criterion is discussed in subsection 3.3.

In earlier discussions, we have kept the fixed removals proportions $p_{1}=p_{2}=\cdots=p_{m-1}=0$ and our main interest was to discuss the optimal choice of inspection times for fixed removals. Moreover, the considered situation was corresponding to the removals which result in the minimum loss of information. At this stage, one may say that situations do arise where the experimenter can control the removals, for example in engineering experiments; although it is true that the removal of units is based on the practical necessity of saving test units or cost. However, one could fix the expected proportion of removals $h$ based on time and cost of the experiment. As soon as $h$ is fixed, the problem of optimum choice in PITI censoring scheme now reduces to the determination of the optimum inspection plan and optimal removal scheme $\left(p_{1}, p_{2}, \ldots, p_{m-1}, p_{m}=1\right)$ where the total proportion of failures $h$ in the experiment is pre-fixed. In this situation, the optimality problem is

$$
\max _{p_{1}, \ldots, p_{m-1}, t_{1},<\cdots<t_{m}} \mathbb{H}(\cdot)
$$

subject to

$$
E\left(r_{1}\right)+E\left(r_{2} \mid r_{1}\right)+\cdots+\left.E\left(r_{m} \mid r_{1}, \ldots, r_{m-1}\right)\right|_{\mu, \sigma}=n \times h .
$$

This scheme is called as generalized optimal spaced (GOS) inspection scheme. Further, we have also studied the effect of removals if these occur in the experiment when inspection times are chosen from Table 1 . So, in this situation, the optimality problem will be

$$
\max _{p_{1}, \ldots, p_{m}} \mathbb{H}(\cdot)
$$

subject to the constraint as given in Eq.(18). The optimum values of $p_{i}$ 's are computed and given in Table 6, under the GOS, OS, OES, and EP inspection schemes for $n=200, m=5$, and $h=0.5,0.6,0.7,0.8$. The first row of the table shows that if the experimenter chooses to use five inspection times and wishes to save $50 \%$ of the $n=200$ units put under test, then the optimal removal scheme will be $11.80 \%, 37.67 \%, 0 \%, 0 \%$, and $100 \%$ removals of the live units at the five consecutive inspection times under GOS inspection scheme. It may be recalled that when we chose the optimum inspection times and studied the effect of variation in the values of $p_{i}^{\prime} s$ the least loss was observed when all $p_{i}^{\prime} s$ was zero except $p_{m}(=1)$. However, from the Table 6 , we see that if we calculate the optimum value of inspection time and removal probability simultaneously then non zero $p_{i}$ s are observed in the solution. Evidently, the optimal censoring plan (i.e choice of inspection time and removal proportion simultaneously) will not be the same if different optimality criteria are used. The entries in the rows titled as OS, OES and EP provides the optimum values of removal proportions when the optimum 
Table 6: Optimal progressive interval Type-I censoring plans under different inspection schemes for some selected failure rates when $\mathrm{n}=200$ and $\mathrm{m}=5$

\begin{tabular}{crcccccr}
\hline $\mathrm{h}$ & Scheme & $p_{1}$ & $p_{2}$ & $p_{3}$ & $p_{4}$ & $p_{5}$ & Relative Efficiency \\
\hline 0.5 & GOS & 0.1180 & 0.3767 & 0 & 0 & 1 & 1 \\
& OS & 0 & 0.4981 & 0 & 0 & 1 & 0.9236 \\
& OES & 0 & 0.0726 & 0.5030 & 0 & 1 & 0.9146 \\
& EP & 0.2904 & 0.1642 & 0 & 0 & 1 & 0.7435 \\
0.4 & GOS & 0 & 0 & 0.4789 & 0 & 1 & 1 \\
& OS & 0.0601 & 0.4194 & 0 & 0 & 1 & 0.9354 \\
& OES & 0 & 0.4554 & 0 & 0 & 1 & 0.9198 \\
& EP & 0.1817 & 0.1931 & 0 & 0 & 1 & 0.7656 \\
0.3 & GOS & 0.0613 & 0.2326 & 0 & 0 & 1 & 1 \\
& OS & 0.0545 & 0 & 0.3115 & 0 & 1 & 0.9402 \\
& OES & 0 & 0.3485 & 0 & 0 & 1 & 0.9233 \\
& EP & 0.2161 & 0 & 0 & 0 & 1 & 0.7847 \\
0.2 & GOS & 0 & 0.1518 & 0.1070 & 0 & 1 & 1 \\
& OS & 0.1085 & 0 & 0.0683 & 0 & 1 & 0.9475 \\
& OES & 0.1627 & 0 & 0 & 0 & 1 & 0.9309 \\
& EP & 0 & 0.2503 & 0 & 0 & 1 & 0.7977 \\
\hline
\end{tabular}

inspection time is pre-calculated and fixed as per optimization criterion discussed in section 3. In this sense, it can be viewed as a result of two-step optimization where we optimize the inspection time at first and then we optimize removal proportion. The last column of Table 6 shows the relative efficiencies of selected inspection scheme with respect to GOS inspection scheme. The resulting relative efficiencies of the OES and OS inspection schemes are $92 \%$ for $h=0.5$ and $95 \%$ for $h=0.2$. But the relative efficiency of EP inspection plan is less than $80 \%$ in all the considered cases which, once again, reveals that the OS and OES inspection scheme are more efficient than the EP inspection scheme. It is also observed that as total removal proportion i.e. $h$ decreases, the relative efficiency approaches to one.

\section{Conclusion}

In this paper, we have contemplated the problem of planning PITI censoring scheme for Rayleigh distribution. It is noted that the inspection times using optimality criterion that minimize Shannon entropy has either highest ARE or close to that relative efficiency which is the highest among all the considered criterion. Moreover, it is also observed that under the equal spacing situation, the Shannon entropy provides the smallest value for the spacing without compromising much in terms of ARE. Therefore, PITI censored plan for Rayleigh distribution must be constructed by using minimum Entropy criterion. In general, the use of the OS inspection scheme to construct the inspection plan reduces the required number of inspections significantly as compared to the EP/OES inspection for achieving the same level of relative efficiency. Thus, for Rayleigh distribution, the OS inspection scheme is more appealing. Moreover, as the choice of inspection times is crucial for the computation of the efficiency of an experiment, hence, the presented work may be productive for future research.

\section{References}

Abd-Elfattah AM, Hassan AS, Ziedan DM (2006). "Efficiency of Maximum Likelihood Estimators under Different Censored Sampling Schemes for Rayleigh Distribution." InterStat, (1), 1-16.

Abdel-Hamid AH, Al-Hussaini EK (2014). "Bayesian Prediction for Type-II ProgressiveCensored Data from the Rayleigh Distribution under Progressive-Stress Model." Journal of Statistical Computation and Simulation, 84(6), 1297-1312.

Aggarwal V (1984). "On Optimum Aggregation of Income Distribution Data." Sankhyā: The Indian Journal of Statistics, Series B, 46(3), 343-355. 
Aggarwala R (2001). "Progressive Interval Censoring: Some Mathematical Results with Applications to Inference." Communications in Statistics - Theory and Methods, 30(8-9), $1921-1935$.

Bai DS, Kim JG, Chun YR (1993). "Design of Failure-Censored Accelerated Life-Test Sampling Plans for Lognormal and Weibull Distributions." Engineering Optimization, 21(3), $197-212$.

Chen DG, Lio YL (2010). "Parameter Estimations for Generalized Exponential Distribution under Progressive Type-I Interval Censoring." Computational Statistics and Data Analysis, 54(6), 1581-1591.

Corana A, Marchesi M, Martini C, Ridella S (1987). "Minimizing Multimodal Functions of Continuous Variables with the Simulated Annealing Algorithm." ACM Transactions on Mathematical Software (TOMS), 13(3), 262-280.

Dey S, Dey T (2014). "Statistical Inference for the Rayleigh Distribution under Progressively Type-II Censoring with Binomial Removal." Applied Mathematical Modelling, 38(3), 974 982.

Ismail AA (2015). "Optimum Partially Accelerated Life Test Plans with Progressively Type-I Interval-Censored Data." Sequential Analysis, 34(2), 135-147.

Kamakura T, Yanagimoto T (1989). "Estimating and Testing Weibull Means Based on the Method of Moments." Technical Report of Department of Statistics, Stanford University, 226, 1-19.

Kaushik A, Pandey A, Maurya SK, Singh U, Singh SK (2017). "Estimations of the Parameters of Generalised Exponential Distribution Under Progressive Interval Type-I Censoring Scheme with Random Removals." Austrian Journal of Statistics, 46, 33-47.

Kaushik A, Singh U, Singh SK (2015). "Bayesian Inference for the Parameters of Weibull Distribution Under Progressive Type-I Interval Censored Data with Beta-Binomial Removals." Communications in Statistics-Simulation and Computation, 46(4), 3140-3158.

Kim C, Han K (2009). "Estimation of the Scale Parameter of the Rayleigh Distribution under General Progressive Censoring." Journal of the Korean Statistical Society, 38(3), 239 - 246.

Kulldorf G (1961). Contributions to the Theory of Estimation from Grouped and Partially Grouped Samples. Wiley, New York.

Lalitha S, Mishra A (1996). "Modified Maximum Likelihood Estimation for Rayleigh Distribution." Communications in Statistics-Theory and Methods, 25(2), 389-401.

Lin CT, Chou CC, Balakrishnan N (2013). "Planning Step-Stress Test Plans under TypeI Censoring for the Log-Location-Scale Case." Journal of Statistical Computation and Simulation, 83(10), 1852-1867.

Lin CT, Wu SJS, Balakrishnan N (2009). "Planning Life Tests with Progressively Type-I Interval Censored Data from the Lognormal Distribution." Journal of Statistical Planning and Inference, 139(1), 54-61.

Lio YL, Chen DG, Tsai TR (2011). "Parameter Estimations for Generalized Rayleigh Distribution under Progressively Type-I Interval Censored Data." Open Journal of Statistics, $\mathbf{1}(02), 46$.

Mousa MA, Al-Sagheer SA (2005). "Bayesian Prediction for Progressively Type-II Censored Data from the Rayleigh Model." Communications in Statistics - Theory and Methods, 34(12), 2353-2361. 
Mousa MA, Al-sagheer SA (2006). "Statistical Inference for the Rayleigh Model Based on Progressively Type-II Censored Data." Statistics, 40(2), 149-157.

Ng HKT, Wang Z (2009). "Statistical Estimation for the Parameters of Weibull Distribution Based on Progressively Type-I Interval Censored Sample." Journal of Statistical Computation and Simulation, 79(2), 145-159.

Rayleigh L (1880). "On the Resultant of a Large Number of Vibrations of the Same Pitch and of Arbitrary Phase." The London, Edinburgh, and Dublin Philosophical Magazine and Journal of Science, 10(60), 73-78.

Seo EH, Kang SB (2007). "AMLEs for Rayleigh Distribution Based on Progressive Type-II Censored Data." Communications for Statistical Applications and Methods, 14(2), 329-344.

Siddiqui MM (1962). "Some Problems Connected with Rayleigh Distributions." Journal of Research of the National Bureau of Standards-D, 60, 167-174.

Sinha SK, Howlader HA (1983). "Credible and HPD Intervals of the Parameter and Reliability of Rayleigh Distribution." IEEE Transactions on Reliability, 2, 217-220.

Wu SJ, Chen DH, Chen ST (2006). "Bayesian Inference for Rayleigh Distribution under Progressive Censored Sample." Applied Stochastic Models in Business and Industry, 22(3), 269-279.

\author{
Affiliation: \\ Arun Kaushik \\ Department of Statistics, Institute of Science \\ Banaras Hindu University \\ Varanasi, India-221005 \\ E-mail: arundevkauhsik@gmail.com \\ URL: http://arun-kaushik.github.io/
}

\section{Austrian Journal of Statistics}

published by the Austrian Society of Statistics

Volume 48

January 2019 http://www.ajs.or.at/

http://www.osg.or.at/

Submitted: 2018-04-11

Accepted: 2018-08-30 